\title{
A rare case presentation of large mucinous ovarian cystadenocarcinoma in a young female
}

\author{
Akansha Singh $^{1 *}$, Ankita Dadhwal ${ }^{1}$, Ashok Verma $^{1}$, Swati Agarwal $^{2}$ \\ ${ }^{1}$ Department of Obstetrics and Gynaecology, ${ }^{2}$ Department of Pathology, Dr. Rajendra Prasad Government Medical \\ College, Kangra, Tanda, Himachal Pradesh, India \\ Received: 02 August 2019 \\ Revised: 17 January 2020 \\ Accepted: 24 January 2020 \\ *Correspondence: \\ Dr. Akansha Singh, \\ E-mail: akkitheonlymab@gmail.com \\ Copyright: (C) the author(s), publisher and licensee Medip Academy. This is an open-access article distributed under \\ the terms of the Creative Commons Attribution Non-Commercial License, which permits unrestricted non-commercial \\ use, distribution, and reproduction in any medium, provided the original work is properly cited.
}

\begin{abstract}
Here authors report a case of large ovarian mucinous cystadenocarcinoma in a young female complicating young pregnancy. Ovarian mucinous adenocarcinoma is a rare ovarian tumour that arises from the surface epithelium of the ovary. A 25-year young female, 9 days post-partum presented to the hospital with complain of abdominal distension. USG finding suggesting large solid cystic mass $36 \times 14 \mathrm{~cm}$ arising from pelvis extending up to epigastrium. MRI pelvis evident of heterogenous hyperintense solid cystic mass lesion giving bunch of grapes appearance with size 24.5 $\times 25 \times 11.5 \mathrm{cms}$ seen in intra peritoneal space extending cranially up to epigastrium and caudally into pelvis giving anterior displacement of uterus. And posterior displacement of bowel loops. A large part of lesion is cystic with solid component with multiple internal echoes.
\end{abstract}

Keywords: Abdominopelvic mass, Mucinous cystadenocarcinoma, Malignant ovarian tumour, Young female

\section{INTRODUCTION}

Mucinous ovarian tumors can be classified as benign, borderline or malignant and can be further classified into invasive and non-invasive. Mucinous cystadenomas are considered benign, non-invasive intraepithelial carcinomas that display cellular atypia but no stroma invasion. ${ }^{1} \quad$ Mucinous ovarian carcinomas present Multicystic tumors with a huge amount of intracellular mucin (present in $\geq 50 \%$ of the cytoplasm) in more than $90 \%$ of tumor cells and contain little extracellular mucin. ${ }^{2}$

Mucinous Ovarian Carcinoma (mOC), however, represents less than five percent of all epithelial ovarian malignancies, and, until recently, the subtleties of this histologic subtype were lost in the larger framework of investigating epithelial ovarian cancers as a whole. ${ }^{3}$ The benign mucinous tumors represent $40 \%$ of all benign ovarian neoplasia, reaching sizes between $15-30 \mathrm{~cm}$ in diameter and they have a 10 years survival rate of $100 \%$. The borderline tumors have a low malignant potential and they have an extra-ovarian spread of about $10 \%$ and the 10 years survival rate is about $60 \%$. The third group is that of the malignant tumor; these tumors are more frequent under the age of 20 years and over 60 years representing $90 \%$ of all malignant ovarian tumors with a 10 years survival rate of about $30-40 \%$.

\section{CASE REPORT}

A 25-year young Indian female presented with a history of abdominal distension of 9 days and lower abdominal pain for 3 days.

Patient had pre-term normal vaginal delivery 9 days back at JLNMC Chamba on $16^{\text {th }}$ July 2019. 
Patient was referred from JLNMC Chamba in view of abdominal-pelvic mass.

Patient was reported to labor room on $25^{\text {th }}$ August 2019 at 1:30 am. She was apparently alright 9 days back, when she started noticing abdominal distension which was acute in onset, rapidly increasing in size associated with pain lower abdomen which was gradual in onset, dull aching, progressively increasing in intensity, nonradiating, no $\mathrm{h} / \mathrm{o}$ associated aggravating and relieving factors. The abdominal distension is associated with loss of appetite for last 3 days.

Patient denies any history of fever, vomiting, night sweats and chills, excessive bleeding per vaginum or alteration of bowel and bladder habits.

\section{Menstrual history}

Patient attainted menarche at 14 years of age. Past menstrual history was unremarkable with flow of 4-5 days every 30 days. At time of presentation, she was having lactational amenorrhoea.

She was married for 4 years with para 2 and live 2 . Patient was not suffering from any chronic disease.

\section{Examination}

General examination revealed normal vital signs. Abdomen was grossly overdistended and tense, distended veins visible, non-tender, cystic, bowel sound was not audible. Uterus noticed as suprapubic bulge around 14 weeks in size, felt separately from mass.

- Per speculum - lochia healthy, os closed

- Cervix and vagina were grossly healthy

- Per vaginum - cervix downward, forward, uterus 1012-week in size firm in consistency non tender, posterior fornix tense and bulging

- Bilateral adnexa could not be made out.

\section{Investigations}

\section{Routine investigation was normal}

Tumour markers - serum LDH - 247 U/L, Beta HCG $4.5 \mathrm{U} / \mathrm{L}, \mathrm{CA} 125$ - $100.4 \mathrm{U} / \mathrm{ml}$, Alfa feto protein - 20.93 ng/ml, CEA - 17.07 ng/ml, CA - 19-9 - 26.09 U/ml.

- USG - large solid cystic mass $36 \times 14 \mathrm{~cm}$ arising from pelvis extending up to epigastrium. A large part of lesion is cystic with solid component with multiple internal echoes. Uterus is bulky and is displaced by the mass anteriorly. Ovaries could not be delineated. Minimal free fluid in pelvis and peri splenic area and Morrison's pouch. B/L hydronephrosis; right sided more than left.

- MRI pelvis - there is evidence of heterogenous hyperintense solid cystic mass lesion giving bunch of grapes appearance with size $24.5 \times 25 \times 11.5 \mathrm{cms}$ seen in intra peritoneal space extending cranially up to epigastrium and caudally into pelvis giving anterior displacement of uterus and posterior displacement of bowel loops. left ovary poorly visualised and right ovary visualised and found normal. Uterus $11.7 \times 12 \times 4.6 \mathrm{cms}$ with ET $-4 \mathrm{~mm}$. Showing above features in Figure 1 and 2.

- There is evidence of free fluid in pelvic region F/S/O? Cystic mesothelioma.

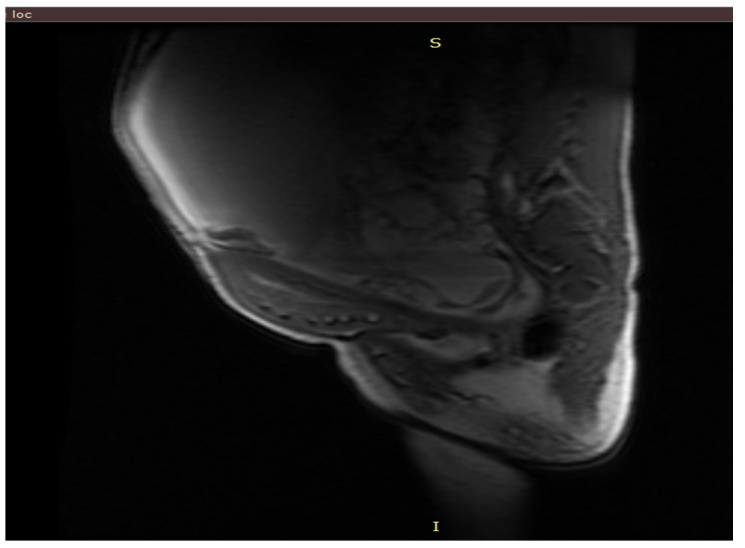

Figure 1: MRI of abdominopelvic mass.

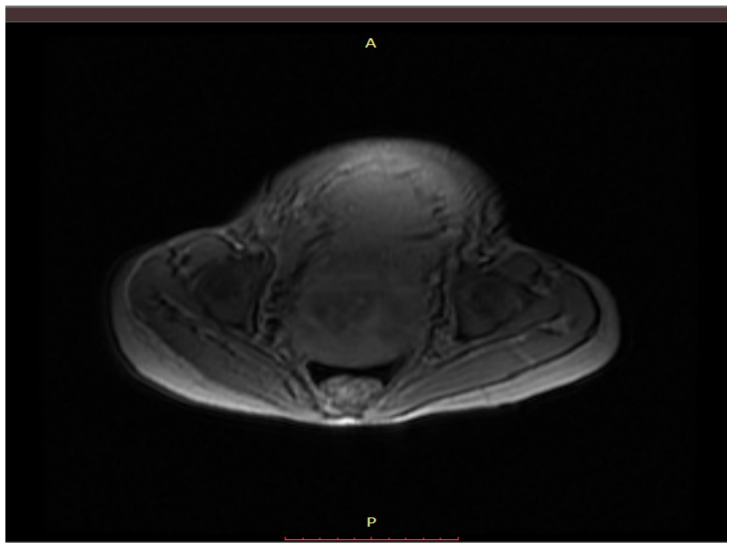

Figure 2: MRI of abdominopelvic mass.

- Patient underwent exploratory laparotomy proceed left salpingo oophorectomy with appendicectomy I/V/O 25 years P2L2 with ovarian mass? Mucinous cystadenoma after histopathology examination report patient underwent re-exploratory laparotomy proceed transabdominal hysterectomy with right side salpingo oophorectomy with intrabdominal exploration with omentectomy.

\section{Intraoperative findings}

On opening abdomen

- No ascites presents 
- Large cystic mass extending from POD to xiphisternum $(40 \times 35 \times 20 \mathrm{~cm})$ arising from left ovary, well encapsulated. Capsule was smooth, no extra capsular spread, multiloculated containing mucinous material

- It was adherent superiorly to omentum laterally to pelvic wall, posteriorly to gut and mesentery and was separated by sharp and blunt dissection

- Capsule ruptured intraoperatively. 4-5 litres of mucinous dark colour material drained after separation of cyst wall from gut, mesentery and abdominal wall, left salpingo oophorectomy done and sent for HPE

- Left fallopian tube thickened and stretched over mass. Rt fallopian tube and Rt ovary and B/L ureter were grossly normal

- Appendix visualized grossly normal, appendicectomy done and sent for HPE

- Caecum, ascending colon and transverse colon and descending colon and sigmoid colon and omentum grossly normal

- $\quad$ Liver, gall bladder and spleen grossly normal

- On cut section - mucinous material present, multiple daughter cyst present.

Areas of hemorrhage and necrosis present, capsule smooth. Showing in Figure 3 and 4.

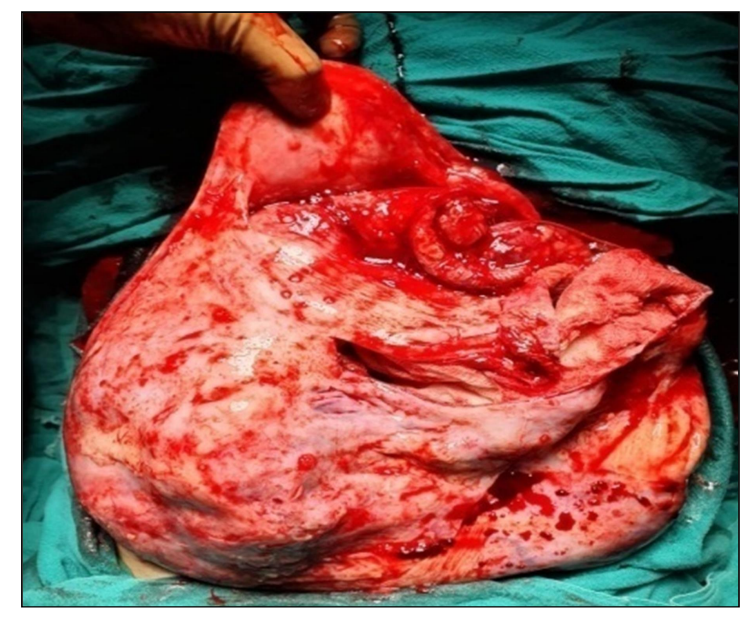

Figure 3: Gross appearance of mass.

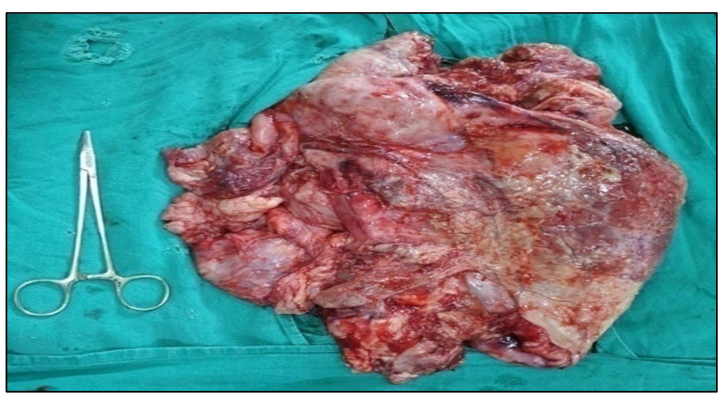

Figure 4: Gross appearance of mass.
Histopathological feature was mucinous cystadenocarcinomas showing in Figure 5 and 6.

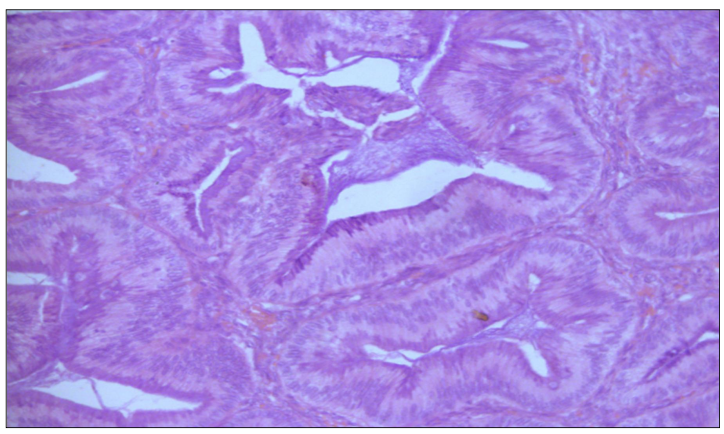

Figure 5: Histopathology of feature of cystadenocarcinoma.

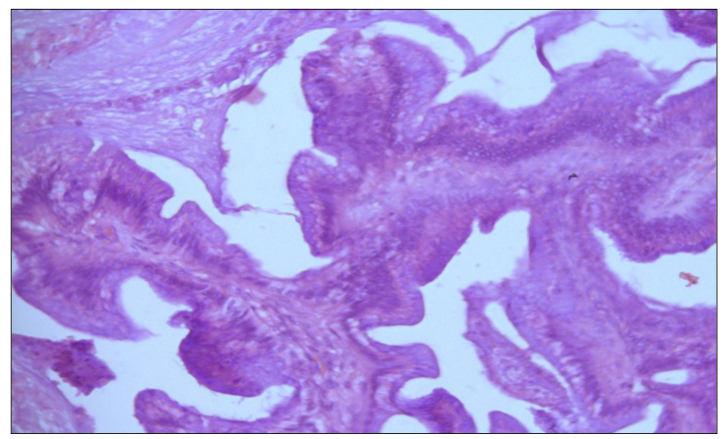

Figure 6: Histopathology of feature of cystadenocarcinoma.

\section{DISCUSSION}

A 25 years para 2-0-0-2 underwent a exploratory laparotomy with proceed left salpingo oophorectomy. The histopathologic work-up showed a mucinous cystadenocarcinoma. After histopathology examination report patient underwent re-exploratory laparotomy proceed transabdominal hysterectomy with right side salpingo oophorectomy with intrabdominal exploration with omentectomy. The postoperative period was uneventful. A giant ovarian cystadenocarcinoma in young patient is a rare finding. Majority of these are benign/borderline tumors with frequency of carcinoma still rarer. Patients mainly present with vague symptoms that are initially ignored by them. This often leads to advancement of disease before the lesion gets diagnosed. Tumor markers and radiology in collaboration serve as an essential tool in the diagnosis of ovarian cancers. CA 125 has been widely used as a marker for epithelial ovarian tumors. Elevated serum CA 125 levels $(>35 \mathrm{U} / \mathrm{mL})$ have been found in more than $80 \%$ of ovarian cancer patients, only $50 \%$ of patients with stage I disease have elevated levels. ${ }^{4}$ It is believed that if levels are initially raised at the time of detection they can be used as a marker for identifying residual or recurrent disease later at follow up. ${ }^{5,6}$ The intra-operative examination of the other ovary 
and surrounding structures is important as it can often lead to upgradation of the stage of the tumor if macroscopic deposits are visualized during surgery. Though the adult staging protocols dictate mandatory lymph node dissections and biopsies of peritoneal surfaces; these procedures are often omitted in young cases unless gross metastatic disease is present ${ }^{7}$ In this patient, right ovary, uterus, fallopian tube and pouch of Douglas were grossly normal no metastatic deposition seen grossly. However, histopathological examination of specimen confirmed the suspicion and upgraded the tumor to stage IC2 by FIGO (International Federation of Gynecology and Obstetrics) guidelines. the significant incidence of bilateral disease, some authors have also recommended a prophylactic wedge biopsy of the uninvolved ovary at the time of initial debulking surgery or biopsy from grossly suspicious foci; however, this was not performed in this case.

A study done by Aggarwal et al, pointed out that low malignant recurrences have been reported more than ten years after initial surgery even in an adult patient population. Therefore, like in adults; young patients should also be kept under close follow up to monitor recurrence which should be treated with another salvage surgery. Various adjuvant regimens ranging from single agent (carboplatin) to multi agent regime have been tried for the treatment of malignant ovarian neoplasms. ${ }^{8}$

Prognosis of ovarian cancers presenting at younger age remains variable and depends on the stage of presentation. Most of the cases reported in the literature have had bad prognosis with almost all the patients dying within five years of detection of the lesion. Prognosis of case too seemed poor.

Epithelial ovarian tumors rarely occur in 25 years of age and are always almost benign. Malignant neoplasms are exceedingly rare however should always be kept in mind especially in cases with raised CA 125 levels suggesting non-germ cell origin. Since signs and symptoms are vague, patient often presents late and at an advanced stage. Management of these cases differ according to staging.

Funding: No funding sources Conflict of interest: None declared Ethical approval: Not required

\section{REFERENCES}

1. Shappell HW, Riopel MA, Smith Sehdev AE, Ronnett BM, Kurman RJ. Diagnostic criteria and behavior of ovarian seromucinous (endocervical-type mucinous and mixed cell-type) tumors: atypical proliferative (borderline) tumors, intraepithelial, microinvasive, and invasive carcinomas. Am J Surg Pathol. 2002;26:1529-41.

2. Kelemen LE, Köbel M. Mucinous carcinomas of the ovary and colorectum: Different organ, same dilemma. Lancet Oncol. 2011;12:1071-80.

3. Frumovitz M, Schmeler KM, Malpica A, Sood AK, Gershenson DM. Unmasking the complexities of mucinous ovarian carcinoma. Gynecol Oncol. 2010;117:491-6.

4. Zanaboni F, Vergadoro F, Presti M, Gallotti P, Lombardi F, Bolis G. Tumor antigen CA 125 as a marker of ovarian epithelial carcinoma. Gynecol Oncol. 1987;28:61-7.

5. Deprest J, Moerman P, Corneillie P, Ide P. Ovarian borderline mucinous tumor in a premenarchal girl: review on ovarian epithelial cancer in young girls. Gynecol Oncol. 1992;45:219-24.

6. Iwasaki M, Taira K, Kobayashi H, Saiga T. Ovarian mucinous cystadenoma of borderline malignancy in a premenarchal girl. J Pediatr Adolesc Gynecol. 2010;23:e119-23.

7. Morowitz M, Huff D, von Allmen D. Epithelial ovarian tumors in children: a retrospective analysis. J Pediatr Surg. 2003;38:331-5.

8. ICON2: randomized trial of single-agent carboplatin against three-drug combination of CAP (cyclophosphamide, doxorubicin, and cisplatin) in women with ovarian cancer. ICON Collaborators. International Collaborative Ovarian Neoplasm Study. Lancet. 1998;352:1571-6.

Cite this article as: Singh A, Dadhwal A, Verma A, Agarwal S. A rare case presentation of large mucinous ovarian cystadenocarcinoma in a young female. Int J Reprod Contracept Obstet Gynecol 2020;9:1746-9. 